Copyright (C 2014 IEEE. Personal use of this material is permitted. Permission from IEEE must be obtained for all other uses, in any current or future media, including reprinting/republishing this material for advertising or promotional purposes, creating new collective works, for resale or redistribution to servers or lists, or reuse of any copyrighted component of this work in other works. 


\title{
Human Gait Phase Recognition Based on Thigh Movement Computed using IMUs
}

\author{
Nimsiri Abhayasinghe* and Iain Murray ${ }^{\dagger}$ \\ Department of Electrical and \\ Computer Engineering \\ Curtin University \\ Perth, Western Australia \\ k.abhayasinghe@curtin.edu.au*, I.Murray@curtin.edu.au ${ }^{\dagger}$
}

\begin{abstract}
Human gait analysis is a major topic in pedestrian navigation and geriatric care. Identifying gait phases is important in using human gait for pedestrian navigation and tracking. Most of existing gait phase identification techniques use multiple sensor modules attached to each section of the lower body. This paper discusses the feasibility of recognizing gait phases using a single inertial measurement unit (IMU) placed in a trouser pocket of the subject. The movement of the thigh is computed by fusing accelerometer and the gyroscopic data gathered from the of the IMU. Experimental results indicated that most of the major gait phases such as Initial Contact, Load Response, Mid Stance, Terminal Stance, Pre-Swing and Swing, can be identified by the movement of one thigh tracked by an IMU. It was also noted that the movement of the offside leg can also be estimated from the fused IMU data. This paper presents a method to recognize all major phases of human stride cycle during walking from movement of one thigh.
\end{abstract}

Keywords-Human gait analysis; inertial sensors; gait phase recognition

\section{INTRODUCTION}

Human gait analysis is important in many applications such as pedestrian navigation and geriatric care. Recognizing gait phases accurately is important for accurate outcomes in gait modeling in pedestrian navigation and locomotion analysis applications. Most of existing inertial measurement unit (IMU) based techniques use inertial sensors attached to one or more sections of the leg to track the movement of the leg or force sensing resistors attached to the sole to detect floor contact or a combination of these [1], [2], [3]. This paper discusses the possibility of recognizing gait phases by estimating the thigh movement from a single thigh mounted IMU (placed in the trouser pocket of the subject). The work discussed in this paper is a part of a research that develops a model for human gait that can be used to estimate the stride length in real time based on the thigh movement for the purpose of vision impaired indoor navigation. Although many state-of-art motion capture systems are available, their usage is limited to laboratory experiments and cannot be used in real-time applications. Hence, discovering new approaches that can be used in real-time gait analysis is important.

Although initial experiments were conducted with people with no vision impairment or physical/mobility disability, observations with vision impaired people were also considered by the authors. The observations discussed in "Thigh Movement with White Cane Usage" subsection indicated that there seems to be an influence on the gait pattern by white cane usage. There are three main types of white cane usages [4]. First is tapping the cane on the ground at position 1 of Fig. 1, raised and arc up to position 2 and tap and vice versa as shown in the figure. The second is sweeping the tip of the cane on the ground from position 1 to position 2 and back. The third, a mix of $1^{\text {st }}$ and $2^{\text {nd }}$ methods, is tapping the cane on the ground at position 1 and sweep the tip on the ground up to position 2 , lift and arc up to position 1. Hence studying the gait pattern with white cane usage is also important in developing the gait model.

Different phases of human gait cycle is discussed in "Human Gait Cycle" section and some existing gait phase recognition techniques are discussed in "Existing Work" section of this paper. The observations made in the experiments and the phases of human gait recognized based on the thigh movement are discussed in "Gait Phase Recognition Based on Thigh Angle" section of this paper and how this is used to estimate stride length in real time is discussed in "Future Work" section.

\section{Human Gait Cycle}

Human gait cycle is defined as the period starting with an initial floor contact of one heel (Initial Contact) to a similar

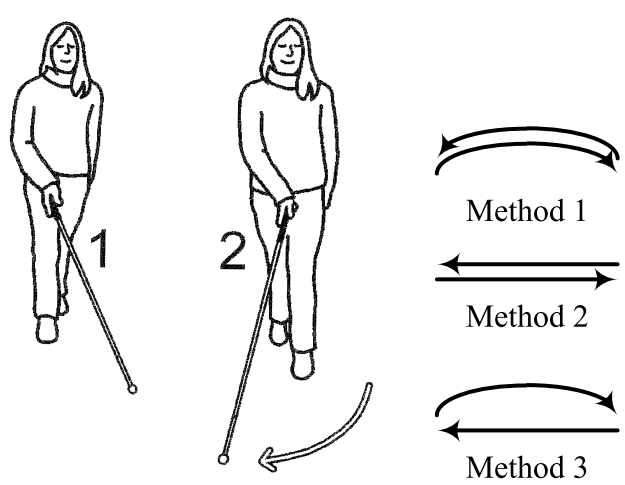

Figure 1. White Cane Usage [4] 


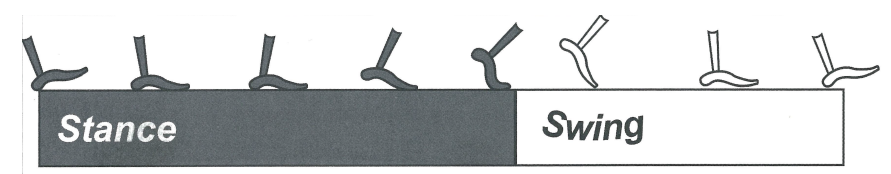

Figure 2. Divisions of the Gait Cycle [5]

posture. A gait cycle can be divided into two main phases: Stance and Swing. During Stance, the reference foot is in contact with the ground while the other foot is in swing, and Swing is where the reference foot is swinging over the ground while the other foot is in contact with the ground [5] as shown in Fig. 2. The Stance phase is further divided into five sub phases: Initial Contact, Loading Response, Mid Stance, Terminal Stance and Pre-Swing whereas the Swing phase is further divided into three sub phases: Initial Swing, Mid Swing and Terminal Swing. Fig. 3 shows the eight sub phases of the human gait cycle.

Initial contact is the moment at which the heel of the reference leg contacts the ground and the other leg is by the end of Terminal Stance phase. The reference foot goes from heel contact to full foot contact during the Loading Response phase while the other leg has the Pre-Swing. By the end of the Loading Response, the body weight is fully transferred to the reference leg (known as single limb support) and during Mid Stance the body weight is tolerated by the reference leg while the other leg is in Initial Swing and Mid Swing phases. During the Terminal Stance, the heel of the reference leg starts moving away from the ground while the toe is still on the ground and the other leg is in Terminal Swing. By the end of the Terminal Stance of the reference leg, the other leg gets the Initial Contact. The last sub phase of Stance, Pre-Swing, is where the reference foot moves further keeping the toe on the ground while the other leg having Loading Response. During Initial Swing, Mid Swing and Terminal Swing phases, the reference leg is swinging above the ground while the other leg undergoes in Mid Stance and Terminal Stance phases. Table I shows the duration of each sub phase of the gait cycle as a percentage of the gait cycle.

\section{EXISTING WORK}

Most of existing real-time gait phase identification techniques use inertial sensors to measure the movement of one or more sections of the leg and force sensing resistors attached to

Table I

Durations of Sub Phases of Human Gait Cycle [5]

\begin{tabular}{|l|c|}
\hline Sub Phase & Interval (of Gait Cycle) \\
\hline \hline Initial Contact & $0 \%-2 \%$ \\
\hline Loading Response & $2 \%-12 \%$ \\
\hline Mid Stance & $12 \%-31 \%$ \\
\hline Terminal Stance & $31 \%-50 \%$ \\
\hline Pre-Swing & $50 \%-62 \%$ \\
\hline Initial Swing & $62 \%-75 \%$ \\
\hline Mid Swing & $75 \%-87 \%$ \\
\hline Terminal Swing & $87 \%-100 \%$ \\
\hline
\end{tabular}

the sole to detect the sole-ground contact. Mijailović et al. [1] have used two accelerometers attached to the thigh and the shank and four force sensing resistors attached to four places of the sole to identify gait phases during walking. They have processed the accelerometers and force sensing resistors separately and reported a maximum gait phase detection with force sensing resistors with an error of $2.7 \%$. However, they have distinguished the Stance phase and Swing phase only.

Wang et al. [2] have used a 3-axis accelerometer attached to an ankle to detect gait phases in their walking pattern classification and walking distance estimation system. They were able to distinguish Initial Contact, Stance phase and Swing phase only with this technique. They have used this information to classify level walking, walking upstairs and down stairs.

A gyroscope based gait phase detection system is discussed in [3] where the authors have used a gyroscope attached to the rear end of the shoe (above the heel) and three force sensing resistors attached to the sole of the shoe. They were able to detect four gait phases only: Heel Strike (Initial Contact), Stance, Heel Off (end of Pre-Swing) and Swing.

All these techniques require sensors be attached to one or more sections of one or both legs, which is not a feasible approach for vision impaired users due to the difficulty of attachment.

\section{Gait Phase Recognition Based on Thigh Angle}

\section{A. Experimental Setup}

In [6] and [7] Abhayasinghe et al. have discussed the possibility of using data of a thigh mounted IMU (placed in the trouser pocket of the subject) to detect the movement of the thigh, accurate step detection and that the gyroscopic data provides sufficient information for gait analysis for pedestrian indoor navigation. This paper discusses how gait phases can be visually identified from the thigh angle waveform and presents the experimental results. IMU data were collected with the involvement of five young males and five young females, all with no vision impairment or physical/mobility disability. Data were recorded while walking on a level surface carrying the device (an Android based smartphone) in one of trouser pockets in upright orientation, screen facing forward. The phone was placed tightly in the pocket so that it remains upright and its movement in the pocket is minimal. A video of the side elevation of one trial was captured so that the estimated movement can be compared with the actual movement.

The IMU was kept vertically in the pocket so that gyroscopic $\mathrm{x}$-axis represent the forward and backward movement of the thigh, which make the computations easy and less resource demanding. Thigh angle was computed by fusing accelerometer and gyroscope data. If gyroscope $\mathrm{x}$-axis reading is small $\left(<0.03 \mathrm{rad} \cdot \mathrm{s}^{-1}\right)$ then the thigh angle is calculated by taking arctan of the ratio $A z / A y$ where $A z$ and $A y$ are the z-axis (perpendicular to the thigh and in forward direction as shown in Fig. Reference axis of IMU data) and y-axis (along the thigh upwards) readings of accelerometer. Gravity is the most significant component of this reading and hence the angle calculated from acceleration is accurate. If gyroscopic $\mathrm{x}$-axis reading 

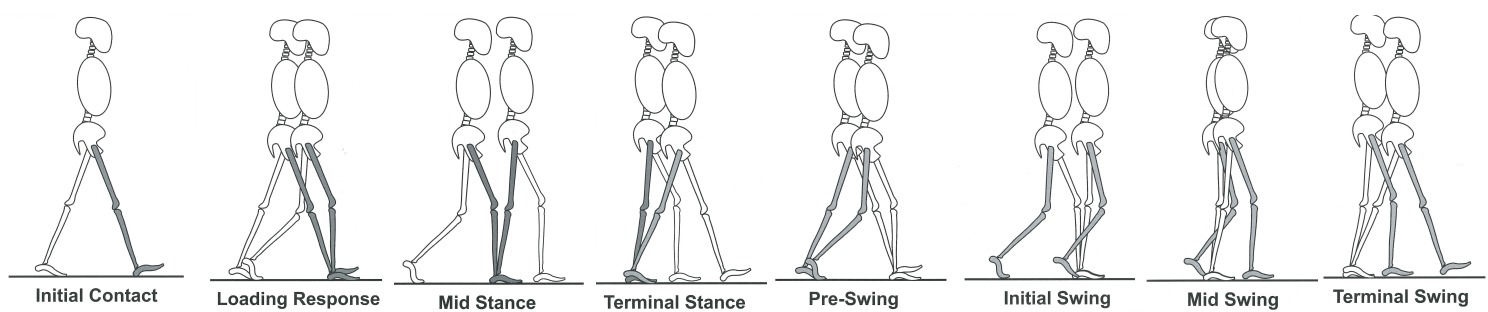

Figure 3. Phases of Human Gait [5]

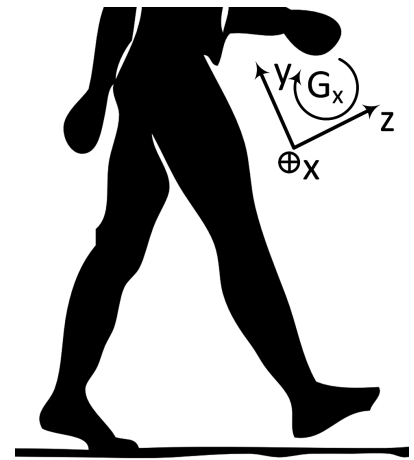

Figure 4. Reference axis of IMU data

is large $\left(\geq 0.03 \mathrm{rad} \cdot \mathrm{s}^{-1}\right)$ then the thigh angle is calculated by integrating gyroscopic $\mathrm{x}$-axis reading because the accelerometer reads body acceleration in addition to gravity, hence angle calculated from accelerometer data is not accurate. By doing the fusion in the aforementioned method, the integration error of gyroscope data is also minimized.

\section{B. Identified Gait Phases}

Out of the eight gait sub phases, the authors were able to distinguish six phases based on the thigh angle. These identified phases are Initial Contact, Loading Response, Mid Stance, Terminal Stance, Pre-Swing and Swing. The three sub phases of Swing: Initial Swing, Mid Swing and Terminal Swing, were not clearly distinguishable in the thigh angle waveform. Fig. 5 shows the computed thigh angle variation with time and the gait phases identified.

The thigh angle is at maximum at Initial contact. During Loading Response, the thigh angle decreases a little and advances again. During Mid Stance, the thigh angle keeps on decreasing down to about $0^{\circ}$ and then starts the Terminal Stance phase where the thigh angle becomes negative. The thigh angle is at its negative peak by the end of the Terminal Stance. During Pre-Swing, thigh angle advances again going a little above $0^{\circ}$ by the end of that phase. The end of the Pre-Swing can be identified by the gradient change occurs by the end which could be due to the fact that the toe moves out of the ground by the end of the Pre-Swing. A gradient change was also observed by the end of the Mid Stance. It could be due to the fact that the other leg transfers from Initial Swing to Mid Swing where that leg goes from back side of the body to front side of the body.
The durations of each sub phase observed in the thigh angle waveform approximately match with the durations given in Table I, which confirms the accuracy of identification of gait phases based on the thigh angle. These durations may be used to double check the detection of each phase.

As discussed in section II, each sub phase of the leg in consideration corresponds to a particular sub phase of the other leg. Hence, proper detection of many phases of one leg only, should be sufficient to analyze the full gait cycle given that there is no physical infirmity to either leg.

\section{Thigh Movement with White Cane Usage}

The next step was to analyze data collected with the involvement of vision impaired subjects while using white cane. Three vision impaired volunteers, two males and one female, participated in this experiment. All participants have no other disability or mobility difficulty. In this experiment too, the IMU was placed vertically in the trouser pocket of the subject and they were asked to walk on a flat surface using the cane.

The plots of computed thigh movement (see Fig. 6 as an example) indicated that the movement is close to the movement of non vision impaired users leading to the fact that the thigh movement can be used to recognize gait phases for vision impaired users. However, some differences were also observed such as slightly different movement during the Loading Response phase and end of Pre-Swing not visible clearly.

It is therefor necessary to undertake further analysis of the gathered data to compare the correlation of thigh movement of vision impaired subjects and non vision impaired subjects and the influence of cane usage the the gait pattern. The authors

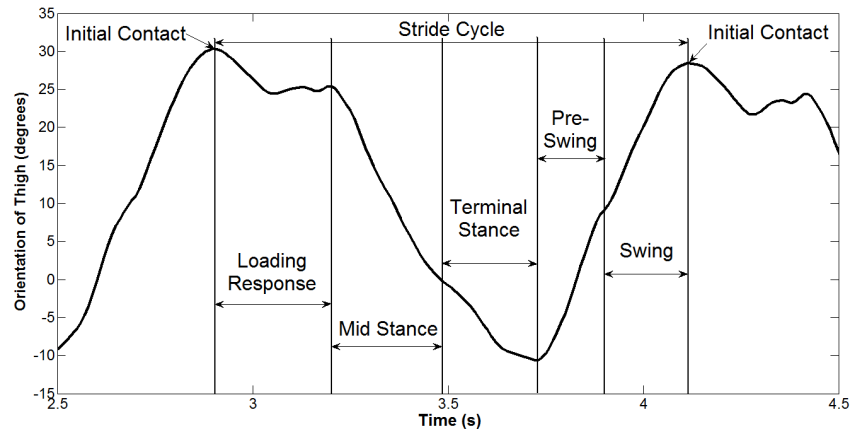

Figure 5. Gait Phases Identified Based on Thigh Angle 


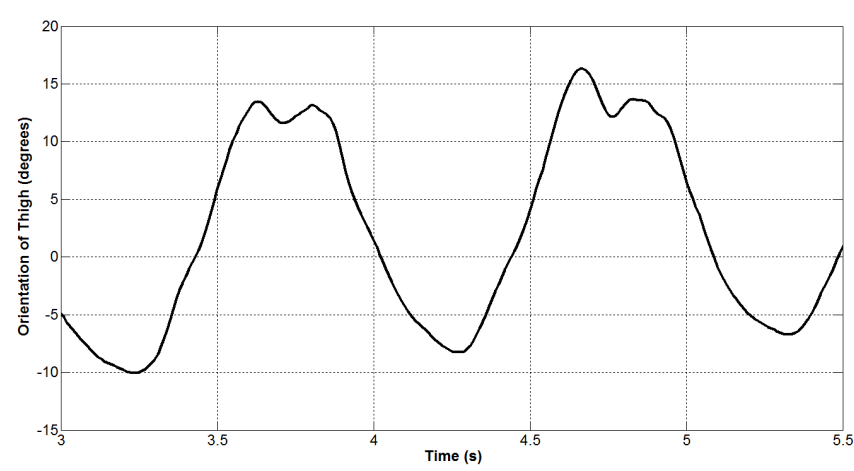

Figure 6. Thigh Angle of a Vision Impaired Subject Using White Cane

have also planned to collect more data with a larger sample size.

\section{FUTURE WORK}

From this preliminary study, it was observed that most of the sub phases of the gait cycle could be identified visually from the plot of computed thigh angle vs. time. However, this waveform has to be mathematically modeled and an algorithm has to be developed to identify gait phases in real-time. Authors are currently working on developing different techniques that can be used to mathematically model this and different signal processing techniques that can be used to detect each phase in real-time. Once this is completed, the algorithm will be first simulated on collected data and then be tested in real-time.

Although state-of-art motion capture systems can be used for laboratory experiments of human gait analysis, they cannot be used in real-time gait analysis which restricts them being used in human navigation applications. However, they can be used as a good reference to estimate the accuracy of the proposed technique. Therefore, the authors have planned to conduct experiments with the participation of both vision impaired and non vision impaired people to collect data from both motion capture system and IMUs simultaneously and compare the results.

A further studies are to be conducted to see if there is any difference in the thigh movement when different footwear are used. In this study, same subject will be asked to walk wearing different types of footwear (like thongs, sandals, sport shoes and boots), which are commonly used by vision impaired users.

As it was observed that there is some influence on the cane usage on the thigh movement, further study on the influence of different types of cane usage on walking pattern should be conducted to see how this influence gait modeling for vision impaired navigation. Further, the experiments have to be extended to different age groups and different motor conditions.

Outcomes of these studies will be helpful in future work of developing a model for human gait based on thigh movement. This model will be used to estimate stride length, particularly for white cane users, in real-time for pedestrian tracking in an indoor/outdoor navigation aid for vision impaired people.

\section{CONCLUSIONS}

This paper presented preliminary analysis results on the possibility of detecting gait phases based on the thigh angle estimated from sensor data collected from a single IMU placed in subject's trouser pocket. Results indicated that six out of eight gait phases can be identified in the thigh angle waveform. Therefore, this approach has significant potential for use in gait phase detection for the purpose of pedestrian navigation. The outcomes can be used in estimating the stride length in realtime based on inertial information of the thigh. However, it was noted that there is an influence to the thigh movement pattern when a vision impaired subject is using a white cane. Hence, further studies are necessary to see the influence of cane usage and different footwear to the gait.

\section{REFERENCES}

[1] N. Mijailović, M. Gavrilović, and S. Rafajlović, "Gait phases recognition from accelerations and ground reaction forces: Application of neural networks," Telfor J., vol. 1, no. 1, pp. 34-36, 2009.

[2] Jeen-Shing Wang, Che-Wei Lin, Y.-T.C. Yang and Yu-Jen Ho, "Walking Pattern Classification and Walking Distance Estimation Algorithms Using Gait Phase Information," IEEE Transactions on Biomedical Engineering, vol.59, no.10, pp.2884-2892, Oct. 2012.

[3] I.P.I. Pappas, M.R. Popovic, T. Keller, V. Dietz and M. Morari, "A reliable gait phase detection system," IEEE Transactions on Neural Systems and Rehabilitation Engineering, vol.9, no.2, pp.113-125, June 2001.

[4] L. Deverell, S. Taylor and J. Prentice, Orientation and Mobility Methods: Techniques for Independent Travel. Victoria, Australia: Guide Dogs Victoria, 2009.

[5] J. Perry and J. M. Burnfield, Gait Analysis: Normal and Pathological Function 2nd Edition. California, US: Slack Incorporated, 2010.

[6] K. Abhayasinghe and I. Murray. (2012, Nov.). "A novel approach for indoor localization using human gait analysis with gyroscopic data," in Third International Conference on Indoor Positioning and Indoor Navigation (IPIN2012) [Online], Sydney, Australia, 2012. Available: http://www.surveying.unsw.edu.au/ipin2012/proceedings/submissions/ 22_Paper.pdf [Mar. 5, 2013].

[7] S. Jayalath, N. Abhayasinghe and I. Murray. (2013, Oct.). "A Gyroscope Based Accurate Pedometer Algorithm," in Forth International Conference on Indoor Positioning and Indoor Navigation (IPIN2013)[Online], Montbéliard, France, 2013, pp. 510-513. Available: http://ipin2013.sciencesconf.org/conference/ipin2013/eda_en.pdf [Nov. 19, 2013]. 